\title{
Fractional statistics description applied to adsorption of alkane binary mixtures in zeolites
}

\author{
M. Dávila, J. L. Riccardo, and A. J. Ramirez-Pastor ${ }^{a)}$ \\ Departamento de Física, Instituto de Física Aplicada, Universidad Nacional de San Luis-CONICET, \\ Chacabuco 917, D5700BWS San Luis, Argentina
}

(Received 30 December 2008; accepted 31 March 2009; published online 6 May 2009)

\begin{abstract}
In the present paper, the multicomponent adsorption of polyatomic species is described as a fractional statistics problem, based on Haldane's statistics. Site exclusion is characterized by a "mutual exclusion matrix" $\mathbf{g}$, which relates to the sizes of the different species and lattice geometry. The adsorption process has been monitored through total and partial isotherms, energy of adsorption and configurational entropy of the adsorbed phase. The thermodynamic functions calculated for a monomer-dimer mixture were applied to describe the adsorption of methane-ethane mixtures in zeolites. In the case of zero lateral interactions, the present approach was compared to the well-known ideal adsorbed solution theory. The results show that the treatment of this complex problem can be significantly simplified if looked up from the new theoretical perspective. (C) 2009 American Institute of Physics. [DOI: 10.1063/1.3124163]
\end{abstract}

\section{INTRODUCTION}

The adsorption of gas mixtures on solid surfaces has received great attention for many decades due to its importance in many technological applications, especially gas separation and purification and catalysis. ${ }^{1-4}$ Particularly, the adsorption of hydrocarbons in zeolites is of considerable practical interest in petrochemical applications due to the unique characteristics of zeolite pores which permit the separation of alkane mixtures on the basis of molecular shape and size. ${ }^{5-7}$ From the theoretical point of view, most of the studies about gas-mixture adsorption are devoted to the adsorption of molecules with single occupancy. ${ }^{8-12}$ However, hydrocarbon molecules adsorbed on solid surfaces should be regarded under the light of a multisite-adsorption model, ${ }^{13}$ in order to properly account for the effects of configurational entropy ( $k$-mer size and flexibility) on the thermodynamics of the adlayer.

The difficulty in the analysis of the multisite statistics is mainly associated with three factors which differentiate the $k$-mers statistics from the usual single-particle statistics. They are the following: (i) no statistical equivalence exists between particles and vacancies; (ii) the occupation of a given lattice site ensures that at least one of its nearestneighbor sites is also occupied; and (iii) an isolated vacancy cannot serve to determine whether that site can ever become occupied. For these reasons, it has been difficult to formulate, in an analytical way, the statistics (and kinetics) of occupation for correlated particles such as dimers. Note that even in the simplest noninteracting dimer case, no exact solution to Langmuir isotherm exists for two or more dimensions.

Over the years, several attempts, successful to varying degrees, have been made to solve the $k$-mer problem. ${ }^{14-20}$

\footnotetext{
a) Author to whom correspondence should be addressed. Electronic mail: antorami@unsl.edu.ar.
}

More recently, a new theory to describe adsorption with multisite occupancy has been introduced, ${ }^{21}$ which incorporates the configuration of the molecule in the adsorbed state as a model parameter. The fractional statistical theory of adsorption (FSTA) of polyatomics is based on a generalization of the formalism of quantum fractional statistics (QFS), proposed by Haldane $e^{22,23}$ as an extended form of Pauli's exclusion principle. The appealing feature of Haldane's statistics or QFS is that a system of interacting particles confined in a finite region of the space can be characterized by a "statistical exclusion parameter," $g$, accounting for the number of states that are excluded from the states spectrum when a particle is added to the system. The relevant range of $g$ in quantum physics is $0<g<1$ being the limiting cases of bosons and fermions statistics, respectively. Although QFS has been interpreted in the context of quantum systems as to describe one-dimensional quantum fluids, fractional quantum Hall effect, quantum thermal conductance, etc., it has been shown that adsorption of structured lattice gases can be treated in the framework of a generalized statistics with an exclusion parameter defined in the range $g>1$. In addition, the advantages of using this simple description as a tool for interpreting polyatomics adsorption data and characterization of the adsorption potential has been shown by analyzing simulation results in lattice and off-lattice gases as well as experimental adsorption isotherm of various adsorbates. ${ }^{24}$

Here, the theory presented in Ref. 21 is extended in order to include adsorption of mixtures of polyatomic species. The new theoretical scheme is applied to study binary alkane mixture adsorption at high pressures, providing a simple model from which experiments may be reinterpreted. The outline of the paper is as follows: In Sec. II we describe the theoretical model. The results and general conclusions are presented in Secs. III and IV, respectively. 


\section{THEORY}

\section{A. Basic formalism: thermodynamic functions}

In what follows, the basis of the mixture adsorption in the framework of the FSTA are introduced. Consider an $m$-component system $\mathbf{N}_{\mathbf{m}}=\left(N_{1}, \ldots, N_{m}\right)$, containing $N_{1}$ molecules of component $1, N_{2}$ molecules of component $2, \ldots, N_{m}$ molecules of component $m$. One isolated component or species $i$ interacting with a regular solid surface confined in a fixed volume $V$ can be represented by an adsorption potential having a spectrum of states with a total number $G_{i}$ of local minima in the space of coordinates necessary to define the adsorption configuration (here the term "species" may apply in general to different adsorbed configurations of a given molecule). Shortly, $\mathcal{G}_{i}$ denotes the set of states for a species $i$ at low density limit, $G_{i}=\# \mathcal{G}_{i}$ being the cardinality of this set (namely, number of equilibrium adsorption states of a single species $i$ in the low density limit). Thus, $\mathbf{G}=\left(G_{1}, \ldots, G_{m}\right)$ represents a vector of coordinates $G_{i}$ 's. Clearly, not all the adsorbed species have the same spectrum of states, hence, in general, $G_{i} \neq G_{j} \forall i \neq j$. In addition, given two species $i$ and $j$, in general the intersection between $\mathcal{G}_{i}$ and $\mathcal{G}_{j}$ is nonempty $\left(\mathcal{G}_{i} \cap \mathcal{G}_{j} \neq \emptyset \forall i, j\right)$. This is clearly the case of two kinds of monomers $i$ and $j$, which can adsorb on the same sites on a surface lattice.

Since all the equilibrium states of the adsorbed species take place in the fixed volume $V$, upon adsorption of a particle of any species $j$ (depending on the typical size and adsorption configuration of the adsorbed species $j$ with respect to the location of the neighboring local minima corresponding to the species $i$ ), a number of states $G_{i 0}\left(\mathbf{N}_{\mathbf{m}}\right)=\sum_{j=1}^{m} g_{i j} N_{j}$ out of $G_{i}$ are excluded (prevented) from further occupation of particles of the species $i$, where the "mutual exclusion parameters" $g_{i j}$ denote the number of states available to the species $i$ excluded by a particle of the species $j$ adsorbed in the volume $V$. Accordingly, given $\mathbf{N}_{\mathbf{m}}$ in the fixed volume $V$, the number of available states for the species $i, d_{i}$, is given by

$$
d_{i}=G_{i}-\sum_{j=1}^{m} g_{i j} N_{j} \quad \text { with } \quad i=1, \ldots, m
$$

The set of $g_{i j}$ 's can be shortly denoted by the "mutual exclusion matrix" $\mathbf{g}$ with range $m^{2}$ and, in matricial notation, $\mathbf{d}=\mathbf{G}-\mathbf{g N}_{\mathbf{m}}$, where $\mathbf{d}=\left(d_{1}, \ldots, d_{m}\right)$.

The set of all available states for the species $i$ is denoted by $\delta_{i}\left(\mathbf{N}_{\mathbf{m}}\right)$ and its cardinality is $d_{i}=\# \delta_{i}\left(\mathbf{N}_{\mathbf{m}}\right)$ [Eq. (1)]. At low density, $\left(N_{1}, \ldots, N_{m}\right) \rightarrow 0, \delta_{i}=\mathcal{G}_{i}$, and $d_{i}=G_{i}$.

Since out of the set of states $\delta_{i}\left(\mathbf{N}_{\mathrm{m}}\right)$, some can be occupied only by the species $i\left[\delta_{i}^{\prime}\left(\mathbf{N}_{\mathbf{m}}\right)\right]$, some by two $\left[\delta_{i j}^{\prime}\left(\mathbf{N}_{\mathbf{m}}\right)\right]$, three $\left[\delta_{i j k}^{\prime}\left(\mathbf{N}_{\mathbf{m}}\right)\right]$, or more different species $\left[\delta_{i j k}^{\prime} \ldots\left(\mathbf{N}_{\mathbf{m}}\right)\right]$, the set $\delta_{i}\left(\mathbf{N}_{\mathrm{m}}\right)$ can be expressed as the conjunction of all nonintersecting $\delta^{\prime}$ 's. In general, the relationship between $\delta^{\prime}$ 's and $\delta$ s depends on the type of problem studied (Sec. II B, this point will be discussed in detail).

In this context, we propose to describe the adsorption of alkane mixtures by means of a generalized statistics with

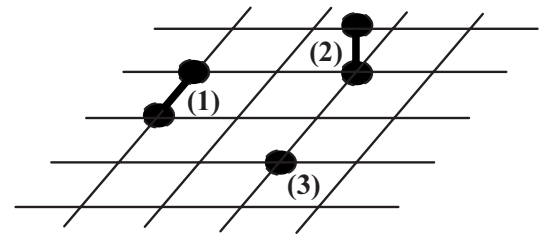

(a)

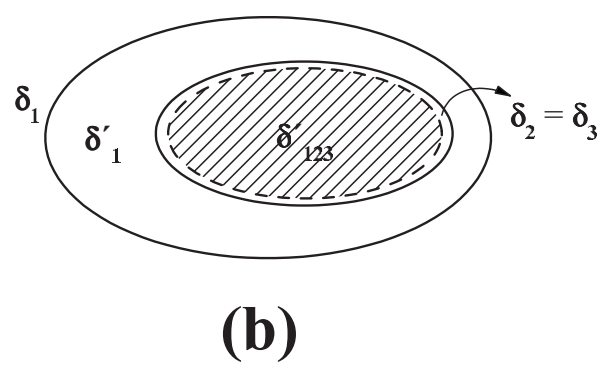

FIG. 1. (a) Schematic representation of the three different adsorption configurations for a monomer-dimer mixture: (1) species 1, dimer lying parallel to the surface (or flat dimer); (2) species 2, dimer adsorbed perpendicular to the surface (or vertical dimer) and (3) species 3, monomer. (b) Snapshot representing the relation between the set of states for a species $i, \delta_{\mathbf{i}}$, and the $\delta^{\prime}$ 's. The image may correspond to the low density limit, where $\left(N_{1}, N_{2}, N_{3}\right) \rightarrow 0, \delta_{i}=\mathcal{G}_{i}$, and $d_{i}=G_{i}$. In this case, $d_{1}=G_{1}=c M / 2$, $d_{2}=G_{2}=M$, and $d_{3}=G_{3}=M$.

$g_{i j} \geq 1$, for which the number of distinguishable configurations of a system characterized by $\left\{N_{1}, \ldots, N_{m}\right\}$ molecules and $d$ state is

$$
\Gamma\left(N_{1}, \ldots, N_{m}, d\right)=\frac{\left(d+\sum_{i=1}^{m} N_{i}-1\right) !}{\left[\Pi_{i=1}^{m} N_{i} !(d-1) !\right]} .
$$

It is tacit that the $d$ states can be occupied by any of the $m$ species.

By assuming $\epsilon_{i}$ is the adsorption energy per particle for the species $i$, the Helmholtz free energy $F\left(N_{1}, \ldots, N_{m}, T, V\right)$ and the partition function $Q\left(N_{1}, \ldots, N_{m}, T, V\right)$ $=\left(\prod_{i=1}^{m} q_{i}^{N_{i}}\right) \Omega\left(N_{1}, \ldots, N_{m}\right) \exp \left(-\beta \sum_{i=1}^{m} \epsilon_{i} N_{i}\right) \quad$ relate through $\beta F=-\ln Q$, where $\beta=1 / k_{B} T$ and $q_{i}$ is the partition function from the internal degrees of freedom of a single molecule of the species $i$ in the adsorbed state. The configurational factor $\Omega\left(N_{1}, \ldots, N_{m}\right)$, can be written as

$$
\begin{aligned}
\Omega\left(N_{1}, \ldots, N_{m}\right)= & {\left[\prod_{i=1}^{m} \Gamma\left(N_{i}, d_{i}^{\prime}\right)\right]\left[\prod_{\langle i, j\rangle_{2}} \Gamma\left(N_{i}, N_{j}, d_{i j}^{\prime}\right)\right] } \\
& \times\left[\prod_{\langle i, j, k\rangle_{3}} \Gamma\left(N_{i}, N_{j}, N_{k}, d_{i j k}^{\prime}\right)\right] \ldots \\
& \times\left[\prod_{\langle i, j, \ldots, l\rangle_{m-1}} \Gamma\left(N_{i}, N_{j}, \ldots, N_{l}, d_{i j \ldots l}^{\prime}\right)\right] \\
& \times \Gamma\left(N_{1}, \ldots, N_{m}, d_{1 \ldots m}^{\prime}\right),
\end{aligned}
$$

where \langle\rangle$_{n}$ represents $n$-uples where all indices are different between them.

The main thermodynamic functions can be obtained from the standard canonical ensemble equations. Thus, 


$$
S=-\left(\frac{\partial F}{\partial T}\right)_{V, N},
$$

and

$$
E=-T^{2}\left(\frac{\partial F / T}{\partial T}\right)_{V, N}
$$

where $S$ and $E$ designate entropy and energy, respectively. Finally, the chemical potential of the adsorbed species $i$, $\mu_{i, \text { ads }}$, can be calculated as

$$
\mu_{i, \mathrm{ads}}=\left(\frac{\partial F}{\partial N_{i}}\right)_{N_{j}^{\prime} \mathrm{s}} .
$$

\section{B. Applications: Lattice model of mixtures of monomers and straight rigid $\boldsymbol{k}$-mers}

In this section, we will calculate explicitly the main thermodynamic functions corresponding to a mixture of monomers and straight rigid $k$-mers adsorbed on a substrate of connectivity $c$. To illustrate these concepts, Fig. 1 shows a typical case, corresponding to a monomer-dimer mixture. This system is of interest for describing the adsorption of methane-ethane mixtures. The lattice-gas model is characterized by three different adsorption states: (1) flat $k$-mers or $k$-mers lying parallel to the surface, and therefore occupying $k$ adjacent sites; (2) upright $k$-mers or $k$-mers adsorbed perpendicular to the surface on single sites; and (3) monomers occupying singles sites. Under these conditions, the problem can be treated as a mixture of three species $(m=3)$, with the following constraints [Fig. 1(b)]:

$$
\delta_{1}=\delta_{1}^{\prime} \cup \delta_{123}^{\prime},
$$

and

$$
\delta_{2}=\delta_{3}=\delta_{123}^{\prime} .
$$

We label flat $k$-mers, upright $k$-mers, and monomers as species 1,2 , and 3 , respectively.

Equation (3) can now be written as

$$
\begin{aligned}
\Omega\left(N_{1}, N_{2}, N_{3}\right) & =\Gamma\left(N_{1}, d_{1}^{\prime}\right) \Gamma\left(N_{1}, N_{2}, N_{3}, d_{123}^{\prime}\right) \\
& =\frac{\Gamma\left(N_{1}, d_{1}\right)}{\Gamma\left(N_{1}, d_{123}^{\prime}\right)} \Gamma\left(N_{1}, N_{2}, N_{3}, d_{123}^{\prime}\right) \\
& =\frac{\Gamma\left(N_{1}, d_{1}\right)}{\Gamma\left(N_{1}, d_{2}\right)} \Gamma\left(N_{1}, N_{2}, N_{3}, d_{2}\right) .
\end{aligned}
$$

In addition,

$$
\begin{aligned}
\mathbf{G} & =(c M / 2, M, M), \\
\mathbf{g} & =\left(\begin{array}{ccc}
\frac{c}{2} k & \frac{c}{2} & \frac{c}{2} \\
k & 1 & 1 \\
k & 1 & 1
\end{array}\right),
\end{aligned}
$$

and, consequently,

$$
\begin{aligned}
\mathbf{d}= & \left(M-\frac{c}{2} k N_{1}-\frac{c}{2} N_{2}-\frac{c}{2} N_{3}, M-k N_{1}-N_{2}-N_{3}, M\right. \\
& \left.-k N_{1}-N_{2}-N_{3}\right),
\end{aligned}
$$

where $M$ is the number of adsorption sites.

Then, from Eqs. (2)-(12)

$$
\Omega\left(N_{1}, N_{2}, N_{3}\right)=\frac{\left[M-N_{1}(k-1)\right] !\left[\frac{c}{2} M-N_{1}\left(\frac{c}{2} k-1\right)-\frac{c}{2} N_{2}-\frac{c}{2} N_{3}\right] !}{N_{1} ! N_{2} ! N_{3} !\left[\frac{c}{2}\left(M-k N_{1}-N_{2}-N_{3}\right)\right] !\left[M-N_{1}(k-1)-N_{2}-N_{3}\right] !} .
$$

Although no interactions between admolecules have been considered in the derivation in order to obtain an explicit analytical isotherm function, the fact that ad-ad interaction may be appreciable can be accounted through a mean-field contribution. The Bragg-Williams approximation is the simplest mean-field treatment for interacting adsorbed particles, even in the case of multisite occupancy. In this context, the canonical partition function $Q\left(N_{1}, N_{2}, N_{3}, T, M\right)$ for a system of $N_{1}$ flat $k$-mers, $N_{2}$ upright $k$-mers, and $N_{3}$ monomers adsorbed on $M$ sites at a temperature $T$, considering nearest-neighbor lateral interaction between adsorbed molecules can be written as

$$
\begin{aligned}
Q\left(N_{1},\right. & \left.N_{2}, N_{3}, T, M\right) \\
= & q_{1}^{N_{1}} q_{2}^{N_{2}} q_{3}^{N_{3}} \Omega\left(N_{1}, N_{2}, N_{3}\right) \\
& \times \exp \left[-\beta\left(\epsilon_{1} N_{1}+\epsilon_{2} N_{2}+\epsilon_{3} N_{3}\right)\right] \\
& \times \exp \left[-\beta\left(w_{11} \bar{N}_{11}+w_{22} \bar{N}_{22}+w_{33} \bar{N}_{33}\right.\right. \\
& \left.\left.+w_{12} \bar{N}_{12}+w_{13} \bar{N}_{13}+w_{23} \bar{N}_{23}\right)\right],
\end{aligned}
$$

where $\quad \exp \left[-\beta\left(w_{11} \bar{N}_{11}+w_{22} \bar{N}_{22}+w_{33} \bar{N}_{33}+w_{12} \bar{N}_{12}+w_{13} \bar{N}_{13}\right.\right.$ $\left.\left.+w_{23} \bar{N}_{23}\right)\right]$ is the mean-field factor, $\bar{N}_{i j}$ the average number of pairs of nearest-neighbor units belonging to the species $i$ and $j$, and $w_{i j}$ represents the nearest-neighbor interaction energy 
between a $i$-molecule and a $j$-molecule. We calculate $\bar{N}_{i j}$ as follows: an $i$-molecule has, on the average (random distribution), $\lambda_{i} \theta_{j}$ nearest-neighbor sites occupied by units belonging to the species $j$, where $\lambda_{i}$ is the number of nearest-neighbor sites of an adsorbed $i$-molecule and $\theta_{j}$ is the partial coverage of each species $j\left(\theta_{1}=k N_{1} / M, \theta_{2}=N_{2} / M\right.$ and $\left.\theta_{3}=N_{3} / M\right)$. Therefore

$$
\bar{N}_{i j}=\frac{1}{2}\left(N_{i} \lambda_{i} \theta_{j}+N_{j} \lambda_{j} \theta_{i}\right),
$$

where the factor $1 / 2$ is inserted to avoid counting each $i j$ pair twice. In addition,

$$
\lambda_{i}=\left\{\begin{array}{cc}
2(c-1)+(k-2)(c-2), & \text { for } i=1 \\
c, & \text { for } i=2 \\
c, & \text { for } i=3 .
\end{array} .\right.
$$

From Eqs. (15) and (16), we can write

$$
\begin{aligned}
& \frac{\bar{N}_{11}}{M}=\lambda_{1} \frac{k N_{1}^{2}}{M^{2}}=\frac{\lambda_{1}}{k} \theta_{1}^{2}, \\
& \frac{\bar{N}_{22}}{M}=c \frac{N_{2}^{2}}{M^{2}}=c \theta_{2}^{2}, \\
& \frac{\bar{N}_{33}}{M}=c \frac{N_{3}^{2}}{M^{2}}=c \theta_{3}^{2}, \\
& \frac{\bar{N}_{12}}{M}=\left(\frac{\lambda_{1}}{2 k}+\frac{c}{2}\right) \frac{k N_{1} N_{2}}{M^{2}}=\left(\frac{\lambda_{1}}{2 k}+\frac{c}{2}\right) \theta_{1} \theta_{2}, \\
& \frac{\bar{N}_{13}}{M}=\left(\frac{\lambda_{1}}{2 k}+\frac{c}{2}\right) \frac{k N_{1} N_{3}}{M^{2}}=\left(\frac{\lambda_{1}}{2 k}+\frac{c}{2}\right) \theta_{1} \theta_{3},
\end{aligned}
$$

and

$$
\frac{\bar{N}_{23}}{M}=c \frac{N_{2} N_{3}}{M^{2}}=c \theta_{2} \theta_{3} .
$$

Accordingly, from Eqs. (13)-(22) and the formalism presented in Sec. II A, the configurational entropy per site $s=S / M$ is given by

$$
\begin{aligned}
\frac{s}{k_{B}}= & {\left[1-\frac{\theta_{1}}{k}(k-1)\right] \ln \left[1-\frac{\theta_{1}}{k}(k-1)\right] } \\
& +\left[\frac{c}{2}-\frac{\theta_{1}}{k}\left(\frac{c k}{2}-1\right)-\theta_{2} \frac{c}{2}-\theta_{3} \frac{c}{2}\right] \\
& \times \ln \left[\frac{c}{2}-\frac{\theta_{1}}{k}\left(\frac{c k}{2}-1\right)-\theta_{2} \frac{c}{2}-\theta_{3} \frac{c}{2}\right]-\frac{\theta_{1}}{k} \ln \frac{\theta_{1}}{k} \\
& -\theta_{2} \ln \theta_{2}-\theta_{3} \ln \theta_{3}-\frac{c}{2}\left(1-\theta_{1}-\theta_{2}-\theta_{3}\right) \\
& \times \ln \frac{c}{2}\left(1-\theta_{1}-\theta_{2}-\theta_{3}\right)-\left[1-\frac{\theta_{1}}{k}(k-1)\right. \\
& \left.-\theta_{2}-\theta_{3}\right] \ln \left[1-\frac{\theta_{1}}{k}(k-1)-\theta_{2}-\theta_{3}\right] .
\end{aligned}
$$

In addition, the total adsorption energy per site $u=E / M$ results

$$
\begin{aligned}
u= & \epsilon_{1} \frac{\theta_{1}}{k}+\epsilon_{2} \theta_{2}+\epsilon_{3} \theta_{3}+\frac{\lambda_{1}}{k} \theta_{1}^{2} w_{11}+c \theta_{2}^{2} w_{22}+c \theta_{3}^{2} w_{33} \\
& +\left(\frac{\lambda_{1}}{2 k}+\frac{c}{2}\right) \theta_{1} \theta_{2} w_{12}+\left(\frac{\lambda_{1}}{2 k}+\frac{c}{2}\right) \theta_{1} \theta_{3} w_{13}+c \theta_{2} \theta_{3} w_{23} .
\end{aligned}
$$

Finally, the chemical potential of the adsorbed species takes the form

$$
\begin{aligned}
y_{1, \text { ads }} \equiv & \beta\left(\mu_{1, \text { ads }}-\epsilon_{1}\right)=(k-1) \ln \left[1-\theta_{1}\left(1-\frac{1}{k}\right)\right] \\
& +\left(\frac{c}{2} k-1\right) \ln \left\{\frac{c}{2}\left[1-\left(\frac{2}{c k}-1\right) \theta_{1}-\theta_{2}-\theta_{3}\right]\right\} \\
& +\ln \frac{\theta_{1}}{k}-\frac{c}{2} k \ln \left[\frac{c}{2}\left(1-\theta_{1}-\theta_{2}-\theta_{3}\right)\right] \\
& -(k-1) \ln \left[1-\left(\frac{1}{k}-1\right) \theta_{1}-\theta_{2}-\theta_{3}\right] \\
& +\beta\left[2 \lambda_{1} \theta_{1} w_{11}+\left(\frac{\lambda_{1}}{2}+\frac{c k}{2}\right) \theta_{1} w_{12}\right. \\
& \left.+\left(\frac{\lambda_{1}}{2}+\frac{c k}{2}\right) \theta_{3} w_{13}\right], \\
y_{2, \text { ads }} \equiv & \beta\left(\mu_{2, \text { ads }}-\epsilon_{2}\right)=\frac{c}{2} \ln \left[1-\left(1-\frac{2}{c k}\right) \theta_{1}-\theta_{2}-\theta_{3}\right] \\
& +\ln \theta_{2}-\frac{c}{2} \ln \left(1-\theta_{1}-\theta_{2}-\theta_{3}\right) \\
& -\ln \left[1-\left(1-\frac{1}{k}\right) \theta_{1}-\theta_{2}-\theta_{3}\right] \\
& +\beta\left[2 c \theta_{2} w_{22}+\left(\frac{\lambda_{1}}{2 k}+\frac{c}{2}\right) \theta_{1} w_{12}+c \theta_{2} w_{23}\right],
\end{aligned}
$$

and

$$
\begin{aligned}
y_{3, \text { ads }} \equiv & \beta\left(\mu_{3, \text { ads }}-\epsilon_{3}\right)=\frac{c}{2} \ln \left[1-\left(1-\frac{2}{c k}\right) \theta_{1}-\theta_{2}-\theta_{3}\right] \\
& +\ln \theta_{3}-\frac{c}{2} \ln \left(1-\theta_{1}-\theta_{2}-\theta_{3}\right) \\
& -\ln \left[1-\left(1-\frac{1}{k}\right) \theta_{1}-\theta_{2}-\theta_{3}\right] \\
& +\beta\left[2 c \theta_{3} w_{33}+\left(\frac{\lambda_{1}}{2 k}+\frac{c}{2}\right) \theta_{1} w_{13}+c \theta_{2} w_{23}\right] .
\end{aligned}
$$




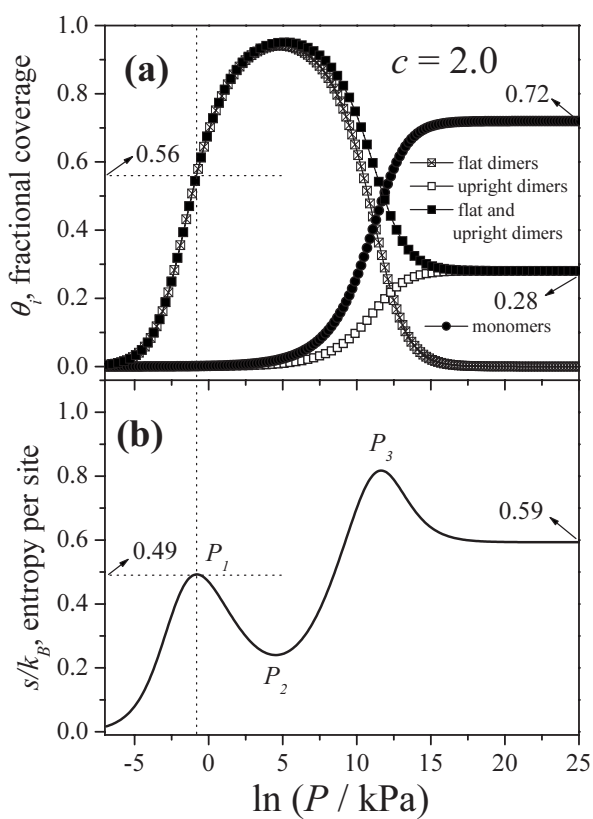

FIG. 2. (a) Partial adsorption isotherms and (b) configurational entropy per site vs surface coverage for an equimolar monomer-dimer mixture on a one-dimensional lattice. Parameter values: $\beta \epsilon_{1}=-21.43, \beta \epsilon_{2}=\beta \epsilon_{3}=-14.48$, $\beta \mu_{d}^{0}=-26.77, \beta \mu_{m}^{0}=-25.83$, and $w_{i j}=0$.

\section{RESULTS}

Now we consider a monomer-dimer mixture, with equimolar amounts of each kind of molecules in gas phase, in two cases: (i) without lateral interactions $w_{i j}=0$, and (ii) with lateral interactions $w_{i j} \neq 0$. The results can be applied to the study of methane-ethane mixture adsorption in silicalite. $^{25}$

At equilibrium, the chemical potential of the adsorbed and gas phase are equal. Then,

$$
\begin{aligned}
& \mu_{1, \mathrm{ads}}=\mu_{d, \mathrm{gas}}, \\
& \mu_{2, \mathrm{ads}}=\mu_{d, \mathrm{gas}},
\end{aligned}
$$

and

$$
\mu_{3, \mathrm{ads}}=\mu_{m, \mathrm{gas}},
$$

where $\mu_{d, \text { gas }}\left(\mu_{m, \text { gas }}\right)$ corresponds to dimers (monomers) in gas phase.

The chemical potentials of each kind of molecule in an ideal gas mixture, at temperature $T$, and pressure $P$, are

$$
\mu_{d, \mathrm{gas}}=\mu_{d}^{0}+k T \ln X_{d} P,
$$

and

$$
\mu_{m, \mathrm{gas}}=\mu_{m}^{0}+k T \ln X_{m} P,
$$

where $\mu_{d}^{0}$ and $\mu_{m}^{0}\left(X_{d}\right.$ and $\left.X_{m}\right)$ are the standard chemical potentials (mole fractions) of dimers and monomers, respectively. In addition,

$$
\mu_{i}^{0}=-k T \ln \left[\left(\frac{2 \pi m_{i} k T}{h^{2}}\right)^{3 / 2} k T\right]
$$

One can work out $\ln P$,

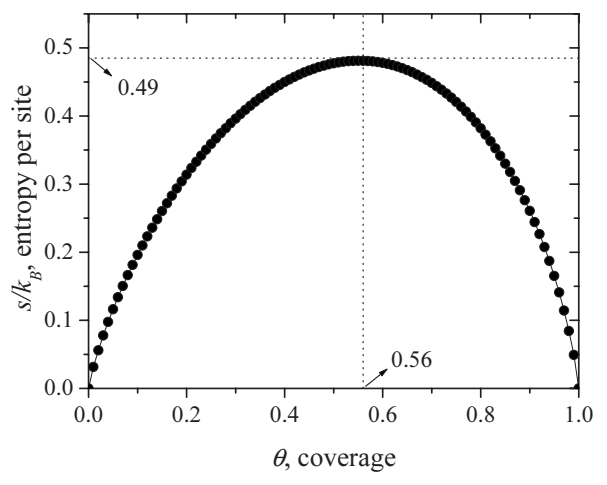

FIG. 3. Configurational entropy per site vs surface coverage for the singlegas adsorption of noninteracting dimers on one-dimensional lattices.

$$
\begin{aligned}
& \ln P=y_{1, \mathrm{ads}}+\beta\left(\epsilon_{1}-\mu_{d}^{0}\right)-\ln X_{d}, \\
& \ln P=y_{2, \mathrm{ads}}+\beta\left(\epsilon_{2}-\mu_{d}^{0}\right)-\ln X_{d},
\end{aligned}
$$

and

$$
\ln P=y_{3, \text { ads }}+\beta\left(\epsilon_{3}-\mu_{m}^{0}\right)-\ln X_{m}
$$

where $y_{1, \text { ads }}, y_{2, \text { ads }}$, and $y_{3 \text {,ads }}$ are given by Eqs. (25)-(27), respectively.

The values of $\beta \mu_{d}^{0}$ and $\beta \mu_{m}^{0}$ were obtained by using Eq. (33) with $m_{m}\left(m_{d}\right)$ equal to the molecular mass of methane (ethane). Thus, $m_{m}=16.04$ uma, $m_{d}=30.07$ uma (where 1 uma $=1.660531 \times 10^{-27} \mathrm{~kg}$ ) (Ref. 26) and, consequently, $\beta \mu_{d}^{0}=-26.77$ and $\beta \mu_{m}^{0}=-25.83$. As in Refs. 10 and $11, T$ is set to $250 \mathrm{~K}$.

Case i: $w_{i j}=0 \forall i, j$. In order to evidence clearly the multisite-occupancy effect associated to the dimers lying parallel to the surface, the adsorption energy assigned to the species 1 is set larger than the adsorption energies corresponding to species 2 and 3. A typical result for this case is shown in Fig. 2, where the values of the adsorption energies per particle were taken from Ref. 10: $\beta \epsilon_{1}=-21.43$ $\left(\epsilon_{1}=-7.4 \times 10^{-20} \mathrm{~J}\right.$ and $\left.T=250 \mathrm{~K}\right) ; \beta \epsilon_{2}=-14.48\left(\epsilon_{2}=-5.0\right.$ $\times 10^{-2} \mathrm{~J}$ and $\left.T=250 \mathrm{~K}\right) ;$ and $\beta \epsilon_{3}=-14.48 \quad\left(\epsilon_{3}=-5.0\right.$ $\times 10^{-20} \mathrm{~J}$ and $T=250 \mathrm{~K}$ ).

The partial adsorption isotherms are shown in Fig. 2(a). Due to the fact that the flat dimers have greater adsorption energy, they begin to adsorb first. As the pressure is increased, the adsorption of monomers and upright dimers starts becoming more energetically favorable, therefore these species start displacing the flat dimers.

On the other hand, the behavior of the configurational entropy as a function of the pressure is reported in Fig. 2(b). The entropy curve presents two peaks and a local minimum, which can be explained as follows: The first peak, at pressure $P_{1}$, occurs when the lattice is occupied by only one of the three species (flat dimers). Then, this singularity can be easily understood by analyzing the dependence on coverage of the configurational entropy of dimers adsorbed on a onedimensional lattice, which is shown in Fig. 3. As it can be observed, the curve in Fig. 3 has a maximum for $\theta \approx 0.56$, being $s / k_{B}(\theta \approx 0.56) \approx 0.49$. These values coincides with those shown in Fig. 2 for the local maximum at $P_{1}$. 


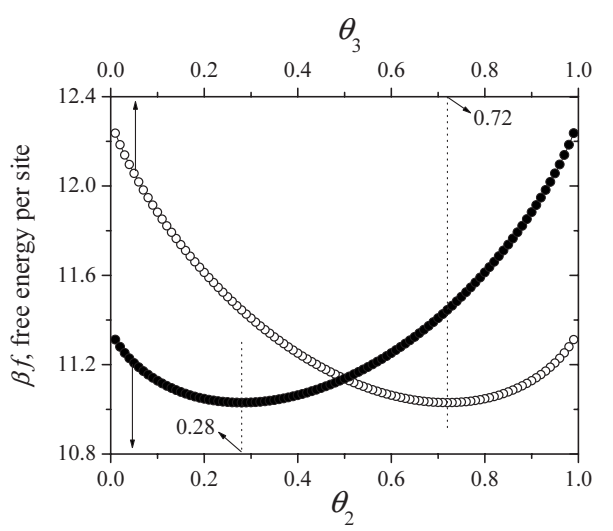

FIG. 4. Free energy per site vs partial coverage of monomers (top axis) and upright dimers (bottom axis) for an equimolar monomer-dimer mixture on a one-dimensional lattice. Energy parameters are as in Fig. 2.

For pressures higher than $P_{1}$, the adsorption of species 1 continues and the configurational entropy diminishes as $\theta_{1}$ is increased, reaching a minimum at pressure $P_{2}$, when the lattice is basically filled with flat dimers. As the adsorption process continues, species 2 and 3 begin to adsorb on the surface, and the entropy increases due to the presence of the three species on the lattice. This process continues until a maximum is reached at pressure $P_{3}$. Then, $s / k_{B}$ decreases monotonically to a constant value for $P \rightarrow \infty$. The limit value of entropy, which will be discussed in details later, is different to zero because there are present two different species on the lattice.

As it can clearly be observed in Fig. 2, the total coverage of dimers (flat dimers+upright dimers) has a maximum and later decreases to a limit value. This behavior is known as adsorption preference reversal (APR) and has been observed in Monte Carlo simulations, mean-field theory, and exact calculations for methane-ethane mixtures adsorption in silicalite. ${ }^{9-11}$ In these papers, the authors show how the competition between two species in presence of repulsive mutual interactions reinforces the displacement of one species by the other and leads to a decreasing in the total coverage.

Our results represent a contribution to the understanding of the phenomenon, showing that if realistic single species adsorption energy values are used when studying mixtures, the APR will appear as a result of the difference in size (or number of occupied sites) between the adsorbed species. Thus, to introduce repulsive lateral interactions in the adsorbate is a fictitious or effective way of taking into account geometric or steric effects by means of energetic arguments. A real description of the displacement of flat ethane by methane and upright ethane should include multisite-occupancy adsorption. A comprehensive study of the APR phenomenon in terms of the difference of size between ethane and methane is under preparation and will be submitted shortly.

In the next, we will discuss the main characteristics of the final state reached for $P \rightarrow \infty$. In this regime $\theta_{1}=0$ and the final state is governed only by species 2 and 3 . In the case studied in Fig. 2, monomers and upright dimers have the same adsorption energy, each molecule occupies one site on the lattice and $X_{d}=X_{m}$. Then, the differences between $\theta_{2}(P \rightarrow \infty)$ and $\theta_{3}(P \rightarrow \infty)$ should be associated with the dif-
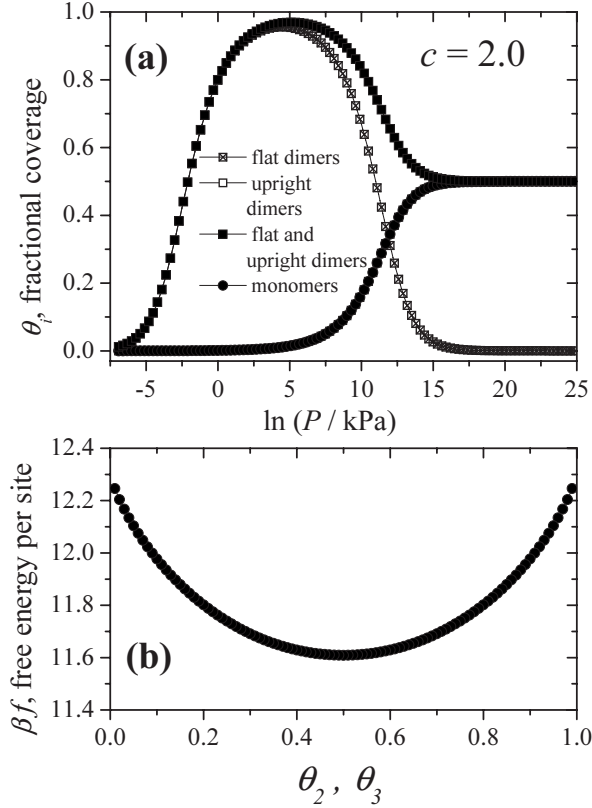

FIG. 5. As in Fig. 2 for $\beta \epsilon_{1}=-21.43, \beta \epsilon_{2}=\beta \epsilon_{3}=-14.48, w_{i j}=0$, and $\mu_{d}^{0}=\mu_{m}^{0}=-25.84$.

ferences between the standard chemical potentials for monomers and dimers. By inspecting Eqs. (25)-(27), we see that the term $\left(\epsilon_{i}-\mu_{i}^{0}\right)$ could be interpreted as an effective adsorption energy corresponding to the $i$ species. Under these considerations, the values of $\theta_{2}(P \rightarrow \infty), \quad \theta_{3}(P \rightarrow \infty)$, and $s / k_{B}(P \rightarrow \infty)$ can be calculated from the minimum of the Helmholtz free energy. The procedure is shown in Fig. 4, where the Helmholtz free energy per site (in $k_{B} T$ units), $\beta f=\beta u-s / k_{B}$, is plotted versus $\theta_{2}$ and $\theta_{3}$. In the calculation of $\beta f$ we use Eq. (23) (with $\theta_{1}=0$ ) and Eq. (24) [with $\theta_{1}=0$ and $\epsilon_{2}$ and $\epsilon_{3}$ equal to the effective adsorption energies $\left(\epsilon_{2}-\mu_{d}^{0}\right)$ and $\left.\left(\epsilon_{3}-\mu_{m}^{0}\right)\right]$. As it can be observed from the figure, the values of $\theta_{2}$ and $\theta_{3}$ in the minimum correspond to the limit values reported in Fig. 2. In addition, introducing these values in Eq. (23), the limit value of the entropy $\left[s / k_{B}(P \rightarrow \infty) \approx 0.59\right]$ is obtained.

As a corroboration of the previous arguments, we repeat the study in Fig. 2, but this time doing $\mu_{d}^{0}=\mu_{m}^{0}$. The results are shown in Fig. 5. Parts (a) and (b) present adsorption isotherms and Helmholtz free energy per site, respectively. In this case, the effective adsorption energies of species 2 and 3 are equal and, consequently, (i) the partial isotherms corresponding to species 2 and 3 coincide; and (ii) $\theta_{2}(P \rightarrow \infty)$ $=\theta_{3}(P \rightarrow \infty)=0.5$ [see final state in Fig. 5(a) and minimum in Fig. 5(b)]. The results obtained reinforce the robustness of the analysis introduced here.

One can do a similar analysis in two dimensions (2D). In this case we present as substrate a lattice with connectivity $c=4$. The fractional coverage and the energy per site are very similar to those in one dimension (1D) because the adsorption energy is the same, and there are not lateral interactions between particles. The entropy is the amount in which one can observe appreciable differences between one dimension and 2Ds (see Fig. 6). In 2D the entropy is higher than in 1D due to the number of available states increases when the connectivity increases. This is shown in the inset of Fig. 6, 


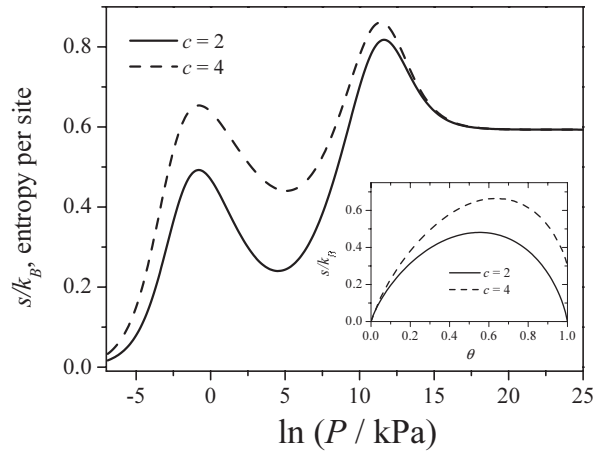

FIG. 6. Configurational entropy per site vs surface coverage for an equimolar monomer-dimer mixture on one-dimensional and two-dimensional lattices as indicated. Parameter values are as in Fig. 2. Inset: configurational entropy per site vs surface coverage for noninteracting dimers adsorbed on one- and two-dimensional lattices as indicated.

where $s / k_{B}$ versus coverage is presented for dimers on 1D and 2D. Both $c=2$ and $c=4$ have the same limit in the configurational entropy because at high pressure there are just monomers and upright dimers in the lattice.

Finally, it is interesting to compare the present approach with the well-known ideal adsorbed solution theory (IAST), developed by Myers and Prausnitz ${ }^{27}$ to predict multicomponent adsorption equilibria. The IAST, which is widely used in engineering models, is based on the assumption that the adsorbed phase can be treated as an ideal solution of the adsorbed components (interested readers are referred to Refs. 7 and 28 for a more complete description of IAST).

In order to compare to the data in Fig. 2, we examine the simplest approximation within IAST. Namely, (1) a mixture of three species is considered: methane molecules, ethane molecules lying parallel to the surface, and ethane molecules adsorbed perpendicular to the surface; (2) each pure component isotherm is given by the Langmuir isotherm; and (3) the values of the adsorption energy parameters are as in Fig. 2. The results are shown in Fig. 7, where the lines represent data from IAST. As it can be observed, IAST does not predict the APR phenomenon for the values of the parameters used. A more refined study, including the multisiteoccupancy effect associated to the ethane molecules lying parallel to the surface, seems to be required to allow the appearance of APR in the framework of IAST. This point will be addressed in future work.

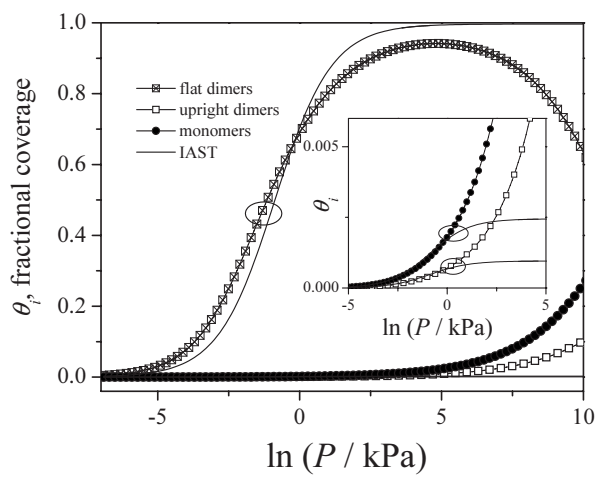

FIG. 7. Comparison between the results in Fig. 2(a) and the corresponding ones obtained from IAST.

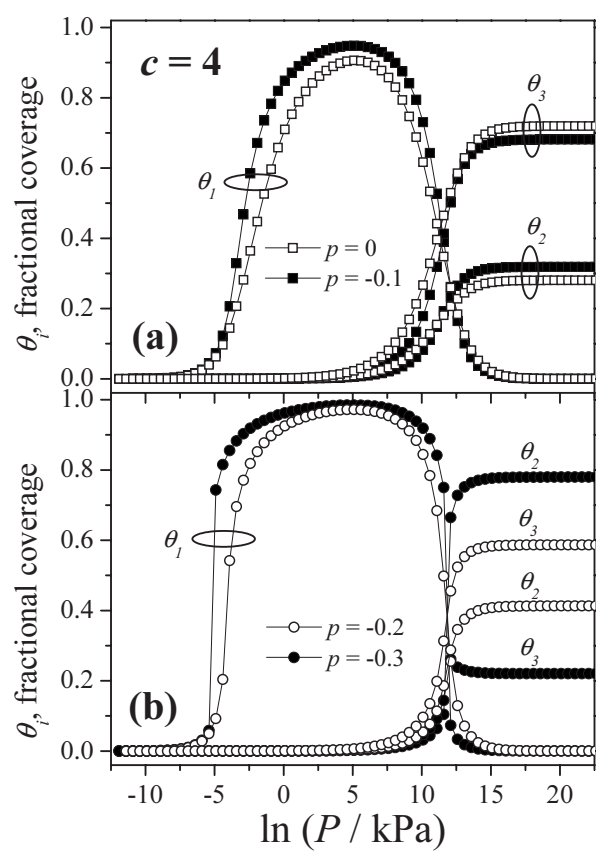

FIG. 8. Partial adsorption isotherms for an equimolar monomer-dimer mixture on a square lattice with $\beta \epsilon_{1}=-21.43, \beta \epsilon_{2}=\beta \epsilon_{3}=-14.48, \beta \mu_{d}^{0}=-26.77$, and $\beta \mu_{m}^{0}=-25.83$. (a) $p=0$ and $p=-0.1$; and (b) $p=-0.2$ and $p=-0.3$.

Case ii: $w_{i j} \neq 0 \forall i, j$. By following the bead segment model, we set the dimer-dimer lateral interaction as the double of the monomer-monomer coupling and the monomer-dimer interaction as the semisum of the monomermonomer and dimer-dimer interactions:

$$
\begin{aligned}
& \beta w_{33}=p, \quad \text { monomer-monomer interaction } \\
& \beta w_{13}=\beta w_{23}=1.5 p, \quad \text { dimer-monomer interaction, } \\
& \beta w_{11}=\beta w_{12}=\beta w_{22}=2 p, \quad \text { dimer-dimer interaction, }
\end{aligned}
$$

where $p$ is a parameter which will be varied.

Figures 8 and 9 present adsorption isotherms and configurational entropy per site, respectively, corresponding to a monomer-dimer mixture with $\beta \epsilon_{1}=-21.43, \beta \epsilon_{2}=-14.48$, $\beta \epsilon_{3}=-14.48, c=4$, and different attractive values of the lateral interactions $p(=0,-0.1,-0.2,-0.3)$.

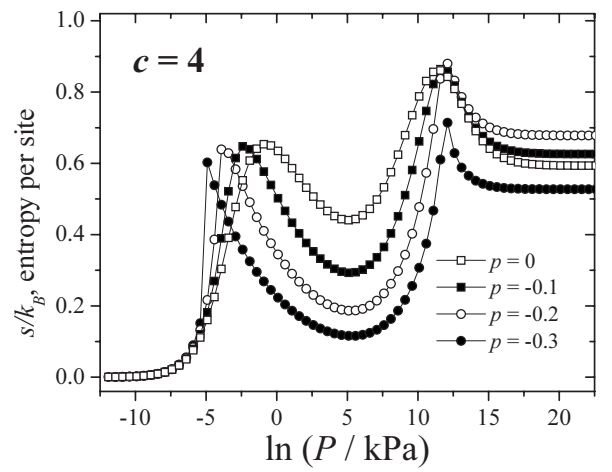

FIG. 9. Configurational entropy per site vs surface coverage for an equimolar monomer-dimer mixture on a square lattice. Parameter values are as in Fig. 8. 


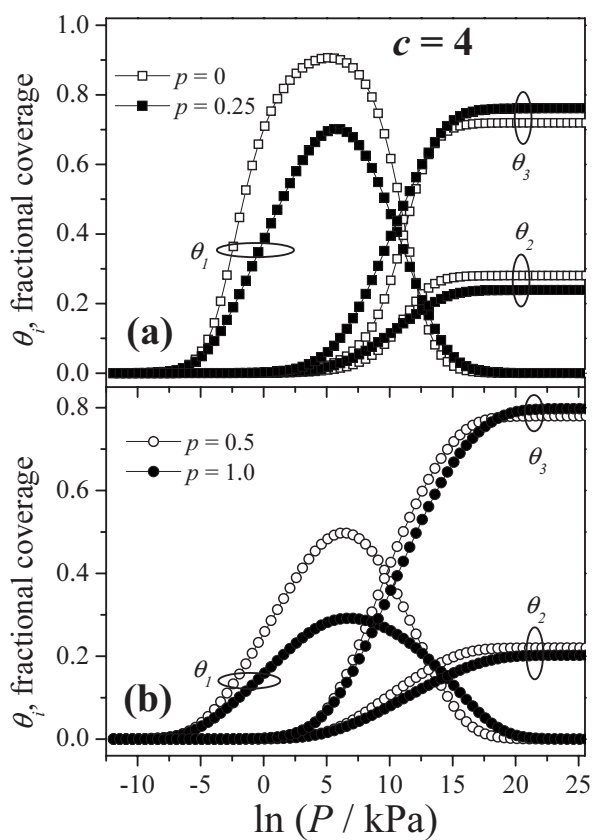

FIG. 10. Same as Fig. 8 for different values of $p$. (a) $p=0$ and $p=0.25$; and (b) $p=0.5$ and $p=1.0$.

As expected, flat dimers isotherms shift to lower values of $\ln P$ and their slope increases as the ratio $\beta w_{33}(p)$ increases, because molecules with attractive units facilitate the adsorption. With respect to species 2 and 3 , the values of the lateral interactions (specifically, $\left|\beta w_{22}\right|>\left|\beta w_{33}\right|$ ) favor the adsorption of upright dimers and, consequently, the characteristics of the final state vary as $|p|$ is increased. Thus, $\theta_{2}(P \rightarrow \infty)<\theta_{3}(P \rightarrow \infty)$ for $|p| \rightarrow 0$ and $\theta_{2}(P \rightarrow \infty)$ $>\theta_{3}(P \rightarrow \infty)$ for $|p|>0.25$.

On the other hand, the curves corresponding to the configurational entropy per site (Fig. 9) present two peaks for all value of $p$. The low-pressure peak, corresponding to flat dimers adsorbed on the lattices, shifts to lower values of the pressure as $|p|$ is increased. This effect is due to the increase in the slope of the flat dimers isotherms. Contrarily, the position of the high-pressure peak due to the presence of the three species on the lattice, remains almost constant as $|p|$ is varied. For pressures between first and second peak, the partial coverage of flat dimers increases with the attractive lateral interactions (see Fig. 8) and, consequently, the minimum of $s / k_{B}$ diminishes as $|p|$ is increased.

The case corresponding to repulsive lateral interactions between the adsorbed particles is shown in Figs. 10 and 11. The values of the parameters used in the figures were $\beta \epsilon_{1}=-21.43, \beta \epsilon_{2}=-14.48, \beta \epsilon_{3}=-14.48, c=4$, and different values of $p(=0,0.25,0.5,1.0)$.

We start with the analysis of the adsorption isotherms (Fig. 10). In contrast to attractive interactions, repulsive lateral interactions do not facilitate the adsorption of the flat dimers and $\theta_{1}$ decreases as $p$ is increased. The decreasing of $\theta_{1}$ is also observed in the curves of the configurational entropy (Fig. 11), where the low-pressure peak disappears for $p>0$. As the pressure is increased, the adsorption of monomers is favored with respect to upright dimers $\left(w_{33}<w_{22}\right)$ and the final value of $\theta_{2}\left(\theta_{3}\right)$ decreases (increases) as $p$ is increased.

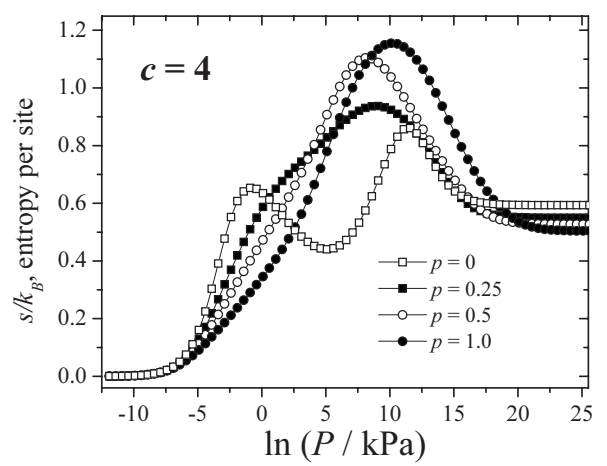

FIG. 11. Configurational entropy per site vs surface coverage for an equimolar monomer-dimer mixture on a square lattice. Parameter values are as in Fig. 10.

The repulsive character of the interactions favors the mixture effect occurring as the three species are present in the lattice and the high-pressure peak in the configurational entropy (see Fig. 11) is reinforced for increasing values of $p$.

Finally, even though the curves corresponding to total coverage of dimers $\left(\theta_{1}+\theta_{2}\right)$ were not shown in Figs. 8 and 10 for clarity, note that the phenomenon of APR also occurs for attractively or repulsively interacting adparticles.

\section{CONCLUSIONS}

The basis of an original theory of multicomponent adsorption of polyatomic species based upon the foundations of Haldane's statistics has been presented. The new formalism provides a close approximation to study mixtures of interacting polyatomics on one- and two-dimensional surfaces with different geometries.

Analysis of adsorption isotherms and configurational entropy per site of the adlayer has allowed a detailed understanding of the adsorption process. This understanding may be useful in developing more general theoretical models and in interpreting experimental adsorption data. In the present case, thermodynamic functions calculated for a monomerdimer mixture were applied to describe the adsorption of methane-ethane mixtures in zeolites. At low pressure the adsorbed phase is almost entirely ethane, but at high-pressure methane displaces ethane reproducing the phenomenon of APR observed in Monte Carlo simulations, mean-field theory, and exact calculations ${ }^{9-11}$ of the system under study. References 9-11 show how the competition between two species in presence of repulsive mutual interactions can be responsible of the displacement of one species by the other. Our results represent a contribution to the understanding of the phenomenon, showing that if realistic single species adsorption energy values are used when studying mixtures, the APR will appear as a result of the difference of size (or number of occupied sites) between the adsorbed species. Thus, to introduce repulsive lateral interactions in the adsorbate is a fictitious or effective way of taking into account geometric or steric effects by means of energetic arguments. A real description of the displacement of flat ethane by methane and upright ethane should include multisite-occupancy adsorption. 
In the case of zero lateral interactions, the present approach was compared to the simplest approximation within IAST. The results revealed that a more refined study, including the multisite-occupancy effect associated to the ethane molecules lying parallel to the surface, seems to be required to allow the appearance of APR in the framework of IAST.

The superiority of the proposed theory relies in its scope (potentially applicable to a wide set of adsorption systems ranging from small polyatomics, hydrocarbons, and perhaps up to light polymers), its simplicity (closed forms of functions), and the smallest number of parameters necessary to account for the surface-molecule/molecule-molecule interactions and the configuration state of the admolecule. A more comprehensive analysis of simulated and experimental systems, necessary to determine the extent of the model to interpret real systems, is in progress.

\section{ACKNOWLEDGMENTS}

This work was supported in part by the CONICET (Argentina) under Project No. PIP 6294, the Universidad Nacional de San Luis (Argentina) under Project No. 322000. and the National Agency of Scientific and Technological Promotion (Argentina) under Project No. 33328 PICT 2005.

${ }^{1}$ D. M. Ruthven, Principles of Adsorption and Adsorption Processes (Wiley, New York, 1984).

${ }^{2}$ R. T. Yang, Gas Separation by Adsorption Processes (Butterworth, London, 1987).

${ }^{3}$ L. K. Doraiswamy, Prog. Surf. Sci. 37, 1 (1991).

${ }^{4}$ W. Rudzinski, W. A. Steele, and G. Zgrablich, Equilibria and Dynamics of Gas Adsorption on Heterogeneous Solid Surfaces (Elsevier, Amsterdam, The Netherlands, 1997).

${ }^{5}$ W. O. Haag, in Zeolites and Related Microporous Materilas: State of the Art 1994, Studies in Surface Science and Catalysis, edited by J.
Weitkamp, H. G. Karge, H. Pfeifer, and W. Hölderich (Elsevier, Amsterdam, The Netherlands, 1994), Vol. 84, p. 1375.

${ }^{6}$ H. B. Abdul-Rehman, M. A. Hasanain, and K. F. Loughlin, Ind. Eng. Chem. Res. 29, 1525 (1990).

${ }^{7}$ B. Smit and T. L. M. Maesen, Chem. Rev. (Washington, D.C.) 108, 4125 (2008).

${ }^{8}$ F. Bulnes, A. J. Ramirez-Pastor, and G. Zgrablich, Adsorpt. Sci. Technol. 23, 81 (2005).

${ }^{9}$ Z. Du, G. Manos, T. J. H. Vlugt, and B. Smit, AIChE J. 44, 1756 (1998).

${ }^{10}$ A. Khettar, S. E. Jalili, L. J. Dunne, G. Manos, and Z. Du, Chem. Phys. Lett. 362, 414 (2002).

${ }^{11}$ L. J. Dunne, G. Manos, and Z. Du, Chem. Phys. Lett. 377, 551 (2003).

${ }^{12}$ L. J. Dunne and G. Manos, Chem. Phys. Lett. 390, 14 (2004).

${ }^{13}$ J. L. Riccardo, F. Romá, and A. J. Ramirez-Pastor, Int. J. Mod. Phys. B 20, 4709 (2006).

${ }^{14}$ L. Onsager, Ann. N. Y. Acad. Sci. 51, 627 (1949).

${ }^{15}$ B. H. Zimm, J. Chem. Phys. 14, 164 (1946).

${ }^{16}$ A. Isihara, J. Chem. Phys. 18, 1446 (1950); 19, 1142 (1951).

${ }^{17}$ P. J. Flory, J. Chem. Phys. 9, 660 (1941); 10, 51 (1942); Principles of Polymers Chemistry (Cornell University Press, Ithaca, NY, 1953); Proc. R. Soc. London, Ser. A 234, 60 (1956).

${ }^{18}$ M. L. Huggins, J. Phys. Chem. 46, 151 (1942); Ann. N.Y. Acad. Sci. 41, 1 (1942); J. Am. Chem. Soc. 64, 1712 (1942).

${ }^{19}$ E. A. DiMarzio, J. Chem. Phys. 35, 658 (1961).

${ }^{20}$ E. A. Guggenheim, Proc. R. Soc. London, Ser. A 183, 203 (1944).

${ }^{21}$ J. L. Riccardo, F. Romá, and A. J. Ramirez-Pastor, Phys. Rev. Lett. 93, 186101 (2004).

${ }^{22}$ F. D. M. Haldane, Phys. Rev. Lett. 67, 937 (1991).

${ }^{23}$ Y. S. Wu, Phys. Rev. Lett. 73, 922 (1994).

${ }^{24}$ F. Romá, J. L. Riccardo, and A. J. Ramirez-Pastor, Ind. Eng. Chem. Res. 45, 2046 (2006).

${ }^{25}$ As is common in the literature, we adopt a "bead segment chain model of the molecules, in which each methyl (bead) group is represented as a $k$-mer unit and coincides in size with one adsorption site on the surface. Under this consideration, we set $k=1,2$ in fitting data corresponding to $C, C 2$, respectively.

${ }^{26}$ CRC Handbook of Chemistry and Physics, 88 th ed., edited by D. R. Lide (CRC, Boca Raton, FL, 2008).

${ }^{27}$ A. L. Myers and J. Prausnitz, AIChE J. 11, 121 (1965).

${ }^{28}$ R. Krishna, S. Calero, and B. Smit, Chem. Eng. J. 88, 81 (2002). 